\title{
TWO-ENDED TOPOLOGICAL GROUPS
}

\section{LEO ZIPPIN}

Introduction. Let $G$ be a locally compact, connected topological group (satisfying the second countability axiom). Let $G^{*}$ be a compact space which contains a dense subset $G^{\prime}$ homeomorphic to the space $G$ and is such that $G^{*}-G^{\prime}$ is totally disconnected. Then, Freudenthal has proved $[1 \text {, Satz } 1 \mathrm{X}, \text { p. 277 }]^{1}$ that the set $G^{*}-G^{\prime}$ consists of at most two distinct points. Actually, Freudenthal's theorem even for topological groups is more general than here stated, and this theorem is an application to group spaces of a wider theory of "ends" of topological spaces. However, we shall quote only so much of Freudenthal's results as are necessary to this paper.

It will be convenient to regard $G^{\prime}$ as identical with $G$ so that $G$ is topologically imbedded in $G^{*}$. We shall call a locally compact, connected group $G$ two-ended if a $G^{*}$ exists such that $G^{*}-G$ consists of two distinct points. The simplest example of such a group is the additive group of reals. Other examples are afforded by the direct product of this group and any compact connected topological group; it is likely that these are the only examples.

The principal objective of this note is the following theorem.

Theorem A. If a locally compact, connected topological group $G$ is two-ended, then $G$ contains a closed subgroup $T$ isomorphic to the group of reals such that the coset-space $G / T$ is compact; moreover, the space $G$ is the topological product of the axis of reals by a compact connected set homeomorphic to the space $G / T$.

1. Definitions. Now let $G$ be two-ended and $G^{*}$ compact, and necessarily connected, such that $G^{*}-G$ consists of a pair of points. We shall denote one of these by $e_{L}$ and the other by $e_{R}$. The space $G^{*}$ is not a group, but we may continue to speak of the group product $f g$ when $f, g \in G \subset G^{*}$. Moreover [1] each $f \in G$ may be regarded as a homeomorphism $f\left(G^{*}\right)=G^{*}$ by the definitions: $f\left(e_{L}\right)=e_{L}$, $f\left(e_{R}\right)=e_{R}, f(g)=f g \in G$ when $g \in G$. To the product of homeomorphisms $f$ and $g$ there corresponds the homeomorphism associated with $f g \in G$. We shall denote by $g K$ (resp. $K g$ ), $g \in G, K \subset G$, the set of points $g k$ (resp. $k g$ ), where $k \in K$.

The two following properties [1] of $G^{*}$ are of great importance in this work.

Presented to the Society, February 26, 1949; received by the editors February 23, 1949.

1 Numbers in brackets refer to the references cited at the end of the paper. 
(1.1) If $g_{n} \in G, n=1,2, \cdots$, and $g_{n} \rightarrow e_{R}$, then $g_{n}^{-1} \rightarrow e_{L}$; and if $g_{n} \rightarrow e_{L}$ then $g_{n}^{-1} \rightarrow e_{R}$.

(1.2) If $K$ is a compact subset of $G$ and $g_{n} \rightarrow e_{R}$ (resp. $e_{L}$ ), then $g_{n} K \rightarrow e_{R}$ (resp. $e_{L}$ ).

In virtue of the known structure of locally compact abelian groups we may, in any locally compact group, distinguish between two classes of elements [2, Lemma 2, p. 96]. One of these is:

(1.3) The class which we shall denote by $C$ consisting of those element of $G$ which are contained in compact subgroups of $G$. For any elements $c \in C$, and any neighborhood $V$ of the identity of $G$, there exists an integer $n$ such that $c^{n} \in V$.

Each element of $G$ not in $C$ generates a subgroup of $G$ isomorphic to the group of integers. For such elements no sequence of distinct powers has any limit in $G$. Now in a two-ended $G$ we may distinguish further:

(1.4) The class $R$ of elements of $G$ such that if $r \in R$ then $r^{n} \rightarrow e_{R}$ (in $G^{*}$ ), and

(1.5) The class $L$ such that $l \in L$ if $l^{n} \in e_{L}$ (in $G^{*}$ ).

It is clear from (1.1) that $R$ and $L$ are each the set of inverses (in $G)$ of the other, are mutually exclusive, and are homeomorphic.

It is another matter, of course, to assert that none of these classes $L, C$, and $R$ is empty, or that they comprise all elements of $G$. This will be shown in the succeeding paragraphs. The assumption that $G$ is two-ended is to be understood throughout; many of the lemmas are not valid in the more general case.

\section{The key lemma.}

Principal Lemma. If $g_{n} \rightarrow e_{R}, g_{n} \in G$, then for all but a finite number, $g_{n} \in R$.

Proof. From the definition of ends [1] it follows at once that there exists a closed compact set $K \subset G$, and open sets $A$ and $B$ of $G^{*}$ such that

$$
\begin{gathered}
G^{*}=A \cup K \cup B, \\
e_{L} \in A, e_{R} \in B, A \cap K=K \cap B=B \cap \bar{A}=A \cap \bar{B}=0 .
\end{gathered}
$$

By (1.1) for almost all $n, g_{n} K \subset B$. Let $g$ denote any $g_{n}$ for which $g K \subset B$. Then $\bar{A} \cap g K=0$.

Now let $A_{0}$ denote the component of $G^{*}-K$ which contains $e_{L}$. Then $A_{0}$ is a maximally connected subset of $A, A_{0}$ is closed in $A$, and $\bar{A}_{0} \cap K$ is not empty since $G^{*}$ is connected $[3$, p. 16]. Let $k$ denote some element of the set $\bar{A}_{0} \cap K$. 


$$
G^{*}=g\left(G^{*}\right)=g A \cup g K \cup g B,
$$

and it is easily seen that $g A_{0}$ is the component of $G^{*}-g K$ which contains $e_{L}=g\left(e_{L}\right)$. Then, since $g K \subset B, \bar{A}_{0} \cap g K=0$ and $\bar{A}_{0} \subset g A_{0}$. Moreover, since $g k \in B, g k \notin \bar{A}_{0}$ and there is some neighborhood $V$ of $k$ such that $g V \cap \bar{A}_{0}=0$. Since $k \in \bar{A}_{0}, V \cap A_{0}$ is not empty. Choose some $a \in V \cap A_{0}$. There is a neighborhood $W$ of the identity such that $W a \subset V$. Then $g W a \subset g V$, and $(g W a) \cap \bar{A}_{0}=0$. Thus we have shown that

$$
\bar{A}_{0} \subset g A_{0}-g\left(W a \cap \bar{A}_{0}\right),
$$

and if we denote $g^{-1}$ by $f$, we may write this as

$$
f \bar{A}_{0} \subset A-W a \cap \bar{A}_{0} \text {. }
$$

Now, for every integer $n$,

$$
f^{n} \bar{A}_{0}=f^{n-1} f \bar{A}_{0} \subset f^{n-1} \bar{A}_{0} \subset \cdots \subset f \bar{A}_{0} \subset A-W a \cap \bar{A}_{0} .
$$

In consequence of this, for every $n, f^{n} a \notin W a$, and therefore $f^{n} \notin W$.

But now it follows from (1.3) that no sequence of powers of $f$ can converge to any element of $G$. On the other hand, since $f^{n} a \subset A$, no subsequence of the points $f^{n} a, n=1,2, \cdots$, can converge to $e_{R} \in B$. Because of (1.2), no subsequence of the set $f^{n}, n=1,2, \cdots$, can converge to $e_{R}$. Finally, then, $f^{n} \rightarrow E_{L}$, and in consequence of this $g^{n} \rightarrow e_{R}$, since $g=f^{-1}$. This proves the lemma.

Corollary. There exists an open set $O^{*} \subset G^{*}, e_{R} \in O^{*}$, such that if $g \in O^{*} \cap G$, then $g \in R$.

This follows from the lemma by the observation that if no such $O^{*}$ existed one could construct a sequence $g_{n} \rightarrow e_{R}$ such that $g_{n} \notin R$, and this would contradict the lemma.

Lemma. If $g \in G$ and for some positive integer $k, g^{k} \in R$, then $g \in R$

Let $Q$ denote an arbitrary open subset of $G^{*}$ containing $e_{R}$. Since $g^{k} \in R$, the sequence $g^{n k} \rightarrow e_{R}, k$ fixed. Therefore for each $m=0,1,2, \cdots, k-1$, the sequences $g^{n k} g^{m}=g^{n k-m} \rightarrow e_{R}$ ( $k$ and $m$ fixed) and there exists an integer $N$ such that for all $n \geqq N$ and all $m=0,1, \cdots, k-1, g^{n k+m} \in Q$. This means, of course, that in the sequence $g, g^{2}, g^{3}, \cdots$, all but a finite number belong to $Q$. Since $Q$ is an arbitrary neighborhood of $e_{R}$, this implies that $g^{n} \rightarrow e_{R}$ and, consequently, $g \in R$.

Corollary. $R$ is open, and $R \cup e_{R}$ is open.

Let $g \in R \subset G$. Then for some integer $k, g^{k} \in O^{*} \cap G$, for the $O^{*}$ of the 
preceding corollary. The set $O^{*} \cap G$ is open and contained in $R$. Then there is a neighborhood $V$ of $g$ such that $V^{k} \subset O^{*} \cap G$. Now for any $f \in V, f^{k} \in O^{*} \cap G \subset R$ and consequently $f \in R$. This establishes that $R$ is open and, since $O^{*} \subset R \cup e_{R}$, it is clear that $R \cup e_{R}$ is open.

3. Another lemma. We have proved now that $R$ is open and not empty. By symmetrical arguments, or by an application of (1.1), the set $L$ is not empty and open. Moreover, in view of the preceding corollary, $e_{L} \cup L \cup R \cup e_{R}$ is an open set whose complement clearly is a closed and compact subset of $G$. Further, if $c$ is an element of this complement, then $c^{n} \notin L \cup R$ for every integer $n$, by a preceding corollary. Then $c \in C$ as defined in (1.3). Since $R \cap L=0$, and since $G$ is connected, it follows that

$$
G=L \cup C \cup R,
$$

where $C$ is closed, compact, not empty. We observe, from the form of (3.1), that $C$ separates $G^{*}$ between $e_{L}$ and $e_{R}$; that is, there is no connected subset of $G^{*}-C$ which contains both $e_{L}$ and $e_{R}$.

Lemma. The identity e of $G$ is a limit of element of $R$.

We know that $C \cap \bar{R}$ is not empty, since $G$ is connected. Let $c \in C$ $\cap \bar{R}$, and let $V$ be an arbitrary neighborhood of the identity. There exists an integer $n$ such that $c^{n} \in V$, by (1.3), and there must exist a neighborhood $W$ of $c$ such that $W^{n} \subset V$. This implies that there are in $V$ elements of the form $r^{n}, r \in W \cap R$. For such $r, r^{n} \in R$. Therefore $R \cap V$ is not empty, and since $V$ is an arbitrary neighborhood of the identity we have established the lemma.

4. One-parameter subgroups. It is our next task to construct in $G$ a one-parameter group $T$ which is closed in $G$. Now if $T$ denotes any one-parameter subgroup of any locally compact group $G$, then by a result of which we have already taken partial advantage $[2$, p. 96] either $T$ belongs to some compact subgroup of $G$, and in that case $T \subset C$, or $T$ is a closed subgroup of $G$. Therefore, to construct in $G$ a subgroup isomorphic to the additive group of reals it suffices to construct a one-parameter group which contains an element not in $C$.

Lemma. $G$ contains a connected abelian group $A$ which contains an element of $R$.

The group $A$ will be constructed with the aid of the preceding lemma. Let $r_{n} \rightarrow e, r_{n} \in R$. Denote by $Q$ an open compact subset of $G$ such that $C \subset Q$, and denote by $A_{n}$ the group generated by $r_{n}$. This 
group is isomorphic to the additive group of integers. Then $A_{n}$ will have elements in $Q$ and also in the complement of $\bar{Q}$, and it is easy to see that there is a subsequence $A_{n_{i}}$ of the sequence $A_{n}$ such that some point of $\bar{Q}-Q$ is in the sequential limit set of the sequence of sets $A_{n_{i}}$. This sequential limit set is the desired group $A$. We have merely sketched the argument because it occurs in substantially the form needed here in $[4$, Lemma 5, p. 112].

Corollary. $G$ contains a subgroup $T$ isomorphic to the additive group of reals.

The closure of the group $A$ of the preceding lemma is a locally compact connected abelian group and is therefore the direct product of a compact subgroup by an $n$-dimensional vector group [5]. Since $\bar{A}$ cannot be compact, containing an element of $R$, it follows that the vector group is not the identity and must contain a subgroup $T$ of the desired property. This is a closed subgroup of $G$, as well as of $\bar{A}$.

5. Conclusion of the proof of Theorem $\mathbf{A}$. We are nearing the end of our task. We know that $G$ contains at least one subgroup isomorphic to the reals. Suppose that $T$ is any such closed one-parameter subgroup. Then the set $e_{L} \cup T \cup e_{R}$ is a closed subset of $G^{*}$ homeomorphic to a simple arc with end points $e_{R}$ and $e_{L}$. Moreover, this is also true of the set $e_{L} \cup_{g} T \cup e_{R}$ for every $g \in G$. Then it follows from the remark following (3.1) that every coset $g T$ contains at least one point of $C$. Let us now consider the coset-space, for definiteness the right coset space $G / T$. Since $T$ is closed in $G, G / T$ may be topologized and is a locally compact topological space [5]. It may assist the reader to remark that an essential feature of this topology is the fact that if $g_{n} \rightarrow g$, where $g, g_{n} \in G$, and if the sequence $g_{n} t_{n}, t_{n} \in T$, has any limit point in $G$ then this limit point is of the form $g t$ for some $t \in T$.

Lemma. The coset-space $G / T$ is compact and homeomorphic to a space $C^{*}$ which is a continuous image of $C$.

Let us denote by $C_{o}$ the set $C \cap g T$, for $g \in C$. This is a closed set. Suppose now that $g_{n} \rightarrow g, g_{n} \in C$, and consider the associated sets $C_{n}=C \cap g_{n} T$. Let $k_{n} \in C_{n}$. Then $k_{n}=g_{n} t_{n}, t_{n} \in T, g_{n} \in C$. Since $C$ is compact, and $k_{n} \in C$, it is an easy consequence that the set of $t_{n}$, $n=1,2, \cdots$, is compact. From this it is clear that any limit point of the set $k_{n}, n=1,2, \cdots$, is of the form $g t \in C_{0}$, for some $t \in T$. Consequently the sets $C_{g}, g \in C$, give rise to an upper semi-continuous decomposition [3] of $C$, and there is a compact space $C^{*}$ which is 
a continuous image of $C, \phi(C)=C^{*}$. For each $c^{*} \in C^{*}$, the set $\phi^{-1}\left(c^{*}\right)$ is some decomposition set $C_{o}=C \cap g T$.

Now it is clear that each point of $C^{*}$ is associated uniquely with a coset $g T$ and therefore with a point of the coset-space $G / T$. Since each coset intersects $C$, this correspondence extends over all of $G / T$ and all of $C^{*}$. From the definition, above, of $C^{*}$ and the topology of $G / T$ it follows easily that this correspondence is a homeomorphism. This establishes the major part of Theorem A.

The fact that the coset-space $G / T$ is homeomorphic to a compact set $C^{*}$ is not alone sufficient to insure that $G$ may be expressed as the topological product of $C^{*}$ and $T$. This would imply, and is implied by, the existence in $G$ of a closed set $\widetilde{C}$ meeting each coset $g T$ in one and only one point. But the fact that $T$ is a closed oneparameter group is enough to insure the existence of such a "crosssection." This was proved by Montgomery and the author [6] in a somewhat more general context, and depends on certain local "sections" of regular families of curves constructed by Whitney. We may conclude, then, that $G$ is representable as the direct product of two closed subsets, the set $T$ and a cross-sectioning set $\tilde{C}$ which is of necessity homeomorphic to $C^{*}$. Since $G$ is connected, it is clear that $\widetilde{C}$ and $C^{*}$ are connected as well as compact. This concludes the proof of Theorem A.

6. An application. As an application of Theorem $A$, let us suppose that a two-ended group $G$ possesses a connected subgroup $H$ which is not compact. Then $\bar{H}$ is locally compact and connected and also two-ended. Therefore, $\bar{H}$ contains a subgroup $T$ isomorphic to the group of reals. Since $T$ is in $G$, it is evident from the preceding section that $G / T$ is compact. All the more, then, $G / \bar{H}$ is compact.

It is an immediate consequence of this remark that if a locally compact connected group $G$ contains a non-compact connected subgroup $H$ such that the coset space $G / \bar{H}$ is not compact, then $G$ cannot be a two-ended group. Therefore, by Freudenthal's theorem which forms the point of departure for this note, $G$ can have only a single "end." This sufficient condition for "one-endedness" generalizes a result due to Freudenthal, appearing in an appendix to his work [7] on the "ends" of discrete-spaces.

\section{REFERENCES}

1. Hans Freudenthal, Neuaufbau der Endentheorie, Ann. of Math. vol. 43 (1942) pp. 261-279. The introduction to this paper contains an account of the earlier supporting work of Freudenthal and others, principally J. de Groot and the present author. 
2. Andre Weil, L'intégration dans les groupes topologique, Actualités Scientifiques et Industrielles, no. 869, Paris, Hermann.

3. G. T. Whyburn, Analytic topology, Amer. Math. Soc. Colloquium Publications, vol. 28, 1942, New York.

4. Deane Montgomery, Connected one dimensional groups, Ann. of Math. vol. 49 (1948) pp. 110-117.

5. L. Pontrjagin, Topological groups, Princeton University Press, 1939.

6. D. Montgomery and L. Zippin, Periodic one-parameter groups, Trans. Amer. Math. Soc. vol. 40 (1936) pp. 24-36.

7. H. Freudenthal, Über die Enden diskreter Räume und Gruppen, Comment Math. Helv. vol. 17 (1944-1945) pp. 1-38.

Queens College 